ISSN: 1679-3013

D.O.I.: $10.5914 / 1679-3013.2013 .0095$

\title{
MICROFITOPLÂNCTON DE ÁGUAS COSTEIRAS ADJACENTES AO PORTO DO RECIFE (PE-BRASIL)
}

\author{
Marcos Antônio Ferreira de MELO ${ }^{1}$ \\ Maria Luise KOENING ${ }^{1}$ \\ José SOUTO Rosa Filho² \\ Rysoaurya Keyla TRAVASSOS ${ }^{1}$ \\ Alex Costa da SILVA ${ }^{1}$
}

Recebido em: julho/2013

Aceito em: agosto/2013

\section{RESUMO}

Neste trabalho foram avaliadas as variações espaciais e temporais do microfitoplâncton em águas costeiras adjacentes ao porto do Recife-PE. As amostras foram tomadas na superfície em sete ocasiões entre maio/2010 e setembro/11 em quatro estações distribuídas ao longo de um transecto paralelo à linha da costa em período de estiagem e chuvoso nos estofos de baixamar de marés de sizígia. As características ambientais variaram amplamente entre os períodos de chuva e estiagem, mas pouco entre estações de coleta. Foram identificados 123 táxons destacando-se o filo Ochrophyta (diatomáceas) com 70,7\%. As espécies dominantes características do

período chuvoso foram Chaetoceros curvisetus Cleve, Coscinodiscus centralis Ehrenberg, Planktothrix agardhii (Gomont) Anagnostidis \& Komárek e Trichodesmium thiebautiiGomont ex Gomonteno período de estiagem destacaram-se Helicotheca tamesis (Shrubsole) M. Ricard e Skeletonema cf. costatum (Greville) Cleve. Não foram registradas diferenças significativas em termos espaciais ou entre períodos climáticos no conjunto de variáveis ambientais, principalmente salinidade e concentrações de nutrientes. De uma forma geral, a comunidade fitoplanctônica variou pouco nos meses de coleta.

Palavras chave: fitoplâncton, variáveis ambientais, zona costeira, sazonalidade

\section{ABSTRACT}

and this work we evaluate the spariation in microphytoplankton in coastal waters adjacent to the portof Recife (Pernambuco State, Brazil). Samples were taken at the surface on seven occasions between May/2010 and September/11 in four stations distributed along a transect parallel to the coast line in the dry and rainy seasons in the spring tides. The environmental characteristics varied widely between periods of rain and drought, but little between sampling stations. One hundred twenty-three taxa were identified, highlighting the phylum Ochrophyta (diatoms) with $70.7 \%$. The dominant species characteristics of the rainy season were Chaetoceros curvisetus Cleve, Coscinodiscus centralis Ehrenberg, Planktothrix agardhii (Gomont) Anagnostidis \& Komarek and Trichodesmium thiebautii Gomont ex Gomont and in the dry season stood out Helicotheca tamesis (Shrubsole) M. Ricard and Skeletonema cf. costatum (Greville) Cleve. There were no significant seasonal or spatial differences in the environmental variables (mainly salinity and nutrient concentrations). The phytoplankton community did not varied significantly, during both dry and rainy seasons.

Keywords: phytoplankton, environmental variables, coastal zone, seasonality

\footnotetext{
1 Departamento de Oceanografia, Universidade Federal de Pernambuco-UFPE, Av. Arquitetura,s/n, Cidade Universitária, CEP 50740-550, Recife - PE, Brasil.

2 Laboratório de Oceanografia Biológica - IG, Universidade Federal do Pará - UFPA, Av. Augusto Corrêa, s/n, Guamá, CEP 66075-110, Belém-PA, Brasil. e-mail: mfmelo37@gmail.com; koening,ufpe.br; souto.rosa@ufpe.br; kekaryt@yahoo.com.br; alex.csilva@ufpe.br
} 


\section{INTRODUÇÃO}

A área costeira do Porto do Recife, Pernambuco, constitui um ecossistema de elevado valor ecológico, econômico e social e apresenta uma série de problemas como degradação natural de seus habitats, declínio da pesca e diminuição da qualidade da água (SANTIAGO et al., 2010). Recebe ainda grande carga de compostos orgânicos e inorgânicos resultante dos aportes naturais e antropogênicos dos efluentes dos estuários dos rios Capibaribe e Beberibe (CPRH, 2001).

O rápido aumento e a diversidade de aportes antropogênicos associados com a urbanização, desenvolvimento costeiro e expansão industrial são as principais causas do declínio da qualidade dos ecossistemas aquáticos (NIXON, 1995; PAERL, 2003), e por consequência alterações na biota.

A comunidade fitoplanctônica de áreas costeiras, composta principalmente por espécies planctônicas oceânicas e neríticas, pode responder de forma direta as alterações nas características do ambiente, através da mudança na composição taxonômica, riqueza, abundância e diversidade (SANTIAGO et al., 2010). A capacidade de responder de forma rápida a flutuações espaciais e temporais das condições ambientais faz com que a comunidade fitoplanctônica seja considerada uma boa bioindicadora de mudanças nos ambientes decorrentes de causas naturais ou do resultado de ações humanas (LIVINGSTON, 2001).

São poucos os trabalhos sobre a comunidade fitoplanctônica realizados próximos a áreas portuárias. Feitosa; Passavante (1991/1993), Feitosa et al. (1999) e Nascimento et al.(2003) conduziram estudos sobre biomassa (clorofila-a) e produção primária na área da Bacia do Pina, considerando a área como eutrófica a hipereutrófica, em decorrência de fortes impactos antrópicos. Koening et al. (1995) estudaram a biomassa fitoplanctônica do estuário do rio Capibaribe e, mais recentemente, Santiago et al. (2010) avaliaram a dinâmica do fitoplâncton no Porto do Recife, concluindo que a área está exposta a impactos devido à proximidade da região metropolitana, e passa por severo processo de eutrofização corroborando os resultados de Travassos et al. (1991) e Eskinazi Leça et al. (1997).

O presente estudo teve como objetivos caracterizar a comunidade microfitoplanctônica na área adjacente ao porto do Recife-PE, descrever as variações espaciais e temporais na estrutura da comunidade e associar as modificações encontradas com alterações naturais e induzidas por ações humanas nas características ambientais.

\section{ÁREA DE ESTUDO}

A cidade do Recife apresenta em seu contexto geomorfológico um variado e bem desenvolvido sistema de zonas úmidas, onde se destaca o complexo estuarino do rio Capibaribe com várias confluências. As principais são entre o rio Beberibe, que vem da Cidade de Olinda e se junta ao rio Capibaribe na bacia de Santo Amaro, a confluência dos rios Tejipió e Jiquiá, que se unem ao Capibaribe e na bacia do Pina, onde todos os canais fluvio-estuarinos convergem para formar a desembocadura (MIRANDA et al., 2010).

O rio Capibaribe ao cortar a planície costeira, onde está a Cidade de Recife, forma um estuário em cuja desembocadura encontra-se o Porto do Recife. O curso do rio Capibaribe é dividido em três partes: o alto e o médio curso, situados no Polígono das Secas, onde o rio apresenta regime temporário (cheio sazonalmente); e o baixo curso que compreende uma faixa de $65 \mathrm{~km}$ que vai do Município de São Lourenço até sua foz, apresentando um regime perene. O rio bifurca-se em dois braços, norte e sul, na jusante da Ponte da Madalena. O braço norte adentra na Bacia de Santo Amaro onde deságua no mar após encontrar o rio Beberibe. O braço sul, devido as várias ações antrópicas, encontra-se praticamente sem vida, percorrendo da Ilha do Retiro até a desembocadura na bacia do Pina.

As águas da bacia do Pina são tipicamente marinhas, com profundidades entre 12-16 metros, enquanto que na Bacia de Santo Amaro, as profundidades não ultrapassam 2,0 metros. A redução da seção batimétrica da bacia do Pina para o rio Capibaribe dificulta a entrada de água das marés de enchente causando uma mistura entre as águas fluviais da bacia hidrográfica do Capibaribe com as águas marinhas. Levando em conta a circulação, o estuário 
do Capibaribe pode ser, portanto, classificado como sendo um estuário de planície, pouco profundo e parcialmente misturado (MONTEIRO et al., 2011).

De acordo com a classificação de Köppen, a área apresenta um clima do tipo As' denominado Tropical quente e úmido, com chuvas de outono e inverno, caracterizando-se por períodos distintos de regime pluviométrico: uma estação seca ou de estiagem, que vai de setembro a fevereiro e uma estação chuvosa de março a agosto. Com temperaturas médias anuais do ar e da água cerca de $25^{\circ} \mathrm{C}$, com mínimo de $24^{\circ} \mathrm{C}$ e máxima $31^{\circ} \mathrm{C}$. A umidade relativa varia entre $80 \%$ a $90 \%$ (ARAGÃO, 2004).

\section{MATERIAL E MÉTODOS}

As coletas foram realizadas bimensalmente em quatro estações ao longo de um perfil paralelo à costa, na área costeira adjacente ao porto de Recife (Fig. 1). As amostras para o estudo do fitoplâncton foram coletadas utilizando-se uma rede de plâncton com abertura de malha de $45 \mu \mathrm{m}$ em arrastos horizontais subsuperficiais, com duração de 10 minutos, a uma velocidade de 2 a 3 nós. Após a coleta, o material foi armazenado em frascos plásticos de 250 $\mathrm{mL}$ e fixado com formol neutro a $4 \%$.

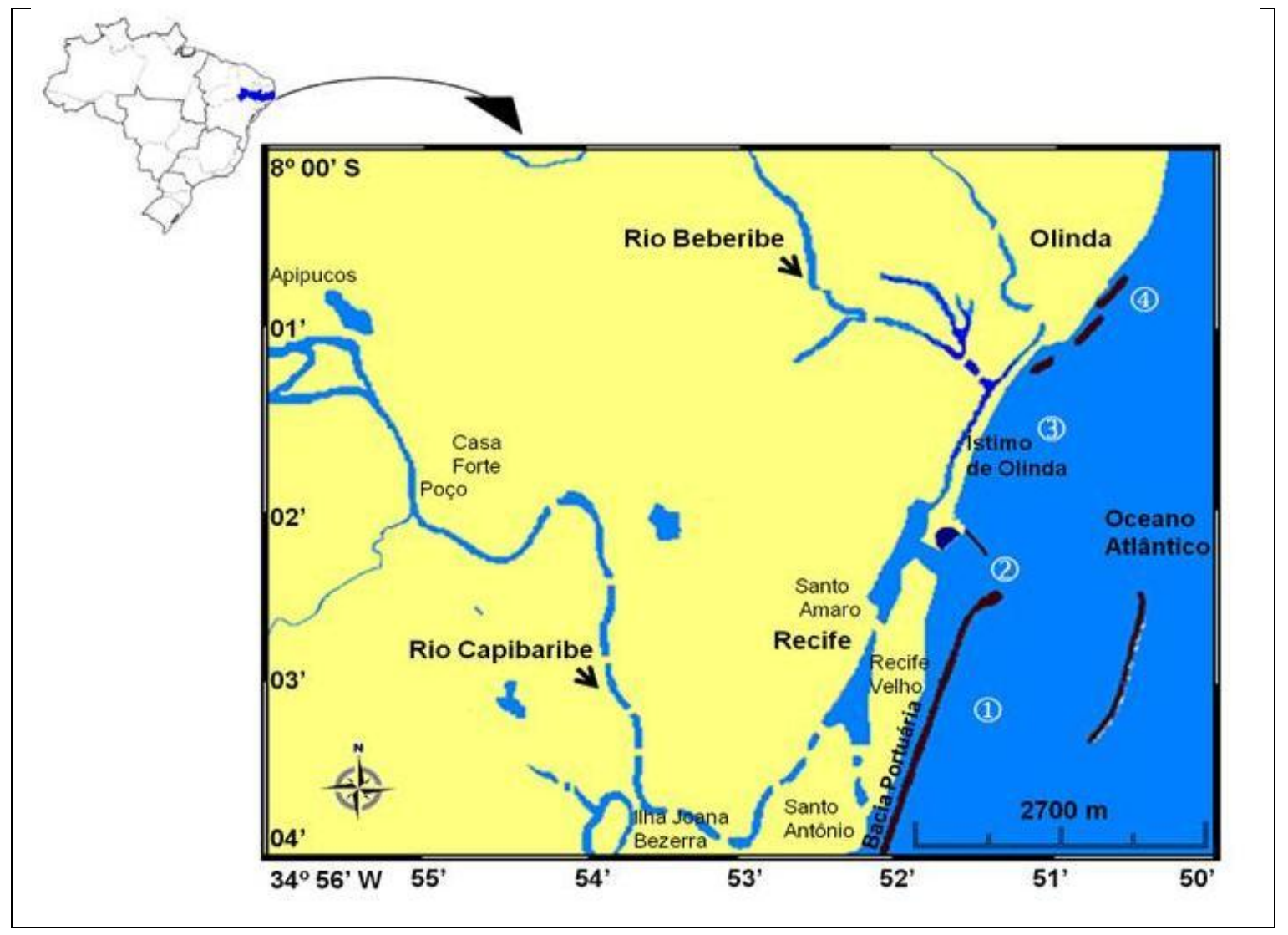

Figura 1 - Localização das estações de coleta (círculos brancos; 1-4) na zona

Simultaneamente, foram aferidos in situ os seguintes parâmetros abióticos: temperatura superficial da água (termômetro de mercúrio graduado em graus Celsius), a profundidade do desaparecimento visual do disco de Secchi (transparência da água). Adicionalmente, foram realizados perfis verticais contínuos de temperatura e salinidade ao longo da coluna d'água em duas campanhas amostrais (novembro/2010 e maio/2011), através de CTD da marca Sea Bird Electronics SBE19plus, equipado com bomba centrífuga e sensores de alta resolução para medições de condutividade (resolução $=0,00004 \mathrm{~S} \mathrm{~m}^{-1}$ ), temperatura (resolução $=0,0003^{\circ} \mathrm{C}$ ) e pressão (resolução $=0,068 \mathrm{db}$ ). A salinidade foi calculada com os algorítmos da escala prática (PSS-178) adotada pela UNESCO, em função dos valores de condutividade elétrica, temperatura e pressão (UNESCO, 1981), para estimar a influência da pluma d'água estuarina $(<34)$ dos rios 
Capibaribe e Beberibe na área de estudo. Foram analisados os registros de salinidade dos dados coletados in situ pelo CTD, nas quatro estações de amostragens, realizadas nas diferentes campanhas amostrais (Fig. 1).

Foram coletadas amostras de água (nas camadas de superfície e fundo) com garrafas van Dorn para posterior determinação do $\mathrm{pH}$ (potenciômetro de bancada Hanna), oxigênio dissolvido (método de Winkler modificado), taxa de saturação de oxigênio (tabelas da UNESCO 1973), concentrações de material em suspensão (Melo et al.,1975), nitrito, nitrato, fosfato (métodos descritos por Strickland e Parsons, 1972) e silicato (Grasshoff et al., 1983).

Para a análise qualitativa do fitoplâncton, as amostras foram homogeneizadas e destas retiradas duas subamostras de $0,5 \mathrm{~mL}$ para a identificação dos táxons até o menor nível taxonômico possível, com auxílio de microscópio óptico e baseada em literatura especializada. Com o auxílio de uma lâmina Sedgwick-Rafte, foi feita uma subamostra para contagem dos táxons e tratamento numérico dos dados. Os critérios de refinamento da identificação e os nomes científicos das espécies foram checados junto ao banco de dados internacional ALGABASE (http://www.algabase.org/).

Foram calculadas para cada amostra os descritores riqueza, considerada como o número total de táxons presentes; abundância relativa, de acordo com Lobo \& Leighton (1986); frequência de ocorrência, seguindo a proposta de Mateucci \& Colma (1982); diversidade, utilizando o índice de Shannon (1948); e, equitabilidade, com o índice de Pielou (1977).

Para identificar modificações espaciais e temporais nas características ambientais e nos descritores da comunidade microfitoplantônica utilizou-se Análise de Variância (ANOVA) bifatorial, considerando os fatores estação de coleta (1-4) e período climático (estiagemchuvoso). Considerou-se período de estiagem os meses de setembro a fevereiro, e chuvoso os meses de março a agosto. Anteriormente a realização das ANOVAs testou-se a normalidade dos dados (Teste de Kolmogorov-Smirinov) e a homocedasticidade das variâncias (Teste de Bartlet). Quando necessário os dados ambientais foram transformados por Log $(x+1)$ e os dados biológicos pela raiz quarta.

As modificações espaço-temporais no conjunto de variáveis ambientais e na estrutura da comunidade fitoplanctônica foram identificadas utilizando-se uma Análise de Similaridade (ANOSIM) bi-fatorial, considerando novamente os fatores estação de coleta e período climático. Para essas análises os dados ambientais foram transformados por Log $(x+10)$ e os biológicos por raiz quarta. A similaridade entre pares de amostras foi calculada pela distância Euclidiana (dados ambientais) e pelo índice de Bray Curtis (dados biológicos). Em todas as análises foi considerado o nível de significância de $5 \%$.

Para identificação dos padrões de variação espacial e temporal das características ambientais e da comunidade fitoplanctônica utilizou-se, respectivamente, a Análise de Componentes Principais (ACP - Dados transformados por Log $(x+1)$ e normalizados) e de agrupamento (Cluster - Dados transformados por raiz quarta, similaridade entre pares de amostras calculada pelo índice de Bray Curtis, amalgamação pelo método das médias aritméticas não ponderadas). Todas as análises foram realizadas empregando-se os programas STATISTICA $^{\circledR} 8.0$ e PRIMER ${ }^{\circledR} 6.0$.

\section{RESULTADOS}

\section{Parâmetros físicos efísico-químicos}

A profundidade na área estudada variou de um mínimo de $3,5 \mathrm{~m}$ na estação 4 em maio/10 a um máximo de $13,5 \mathrm{~m}$ na estação 2 em novembro/2010. Variação significativa $(p=0,038)$ apenas entre estações, particularmente a estação 4 , que foi significativamente mais rasa que as demais.

Os valores de transparência da água resultantes da profundidade de desaparecimento do disco de Secchi apresentaram valor mínimo de 0,50 $\mathrm{m}$ na estação 2 registrado no mês de julho/2010 e máximo de 6,0 m na estação 4 em novembro/2010, ambos na camada superficial. Não houve variação significativa espacial $(p=0,066)$ ou sazonal $(p=0,187)$, ainda que os valores tenham sido sempre maiores no período de estiagem. 
A salinidade apresentou variação sazonal significativa $(p=0,043)$, com valores que oscilaram entre o mínimo de 14,80 na estação 2 superfície em julho/11 e o máximo de 37,16 nas estações 3 e 4 em novembro/10 na camada de fundo, mas não variaram significativamente entre estações de coleta $(p=0,254)$. Durante a campanha amostral de maio/2011 foram observados na superfície (na profundidade de um metro) a presença de uma pluma d'água de baixa salinidade nas quatro estações de coleta, com valores de salinidade variando entre 16 34.

Durante a campanha amostral de novembro/2010 houve a presença de pluma d'água estuarina de baixa salinidade (< 34) nas estações 2 e 3, localizada em torno de 0,5 m de profundidade abaixo da linha superficial d'água. Nas estações 1 e 4 não houve presença de uma massa d'água de baixa salinidade na superfície da coluna d'água, mas a presença de um pico de alto valor de salinidade ( $>37$ ) em torno de um metro de profundidade.

Em novembro/2010 foram observados valores superficiais de temperatura em torno de $28,6{ }^{\circ} \mathrm{C}$ nas quatro estações de coleta (estação 1, 2, 3 e 4). Durante a campanha amostral de maio/2011 foram observados valores máximos de temperatura (em torno de $28,3^{\circ} \mathrm{C}$ ) na profundidade de $2 \mathrm{~m}$ nas estações 3 e 4 .

O oxigênio dissolvido apresentou concentrações mais baixas no período chuvoso com um mínimo de $1,73 \mathrm{ml} . \mathrm{L}^{-1}$ em julho/10 na estação 2 na superfície. No período de estiagem foi registrado o valor máximo de $7,78 \mathrm{ml} . \mathrm{L}^{-1}$ também na estação 2 em setembro/11, na camada de fundo com forte variação no período climático e espacial, ainda que apenas a variação entre estações tenham sido significativas $(p<0,001)$.

Todos os valores de pH foram alcalinos, com o mínimo de 7,96 na estação 3 (camada superficial) e máximo de 8,94 nas estações 2 e 4, ambos em julho/2011, na superfície.O pH não variou significativamente entre os períodos climáticos $(p=0,056)$ e estações de coleta $(p=0,327)$.

De um modo geral, o material particulado em suspensão não apresentou variação significativa espacial $(p=0,403)$ ou entre os períodos climáticos $(p=0,827)$. Os valores variaram entre $2,80 \mathrm{mg} . \mathrm{L}$ em março/11 na estação 4 e $64,00 \mathrm{mg} . \mathrm{L}$ na estação 3 em julho/10, ambas na camada de fundo.

Para os teores de nitrito foram registrados valores abaixo do limite de detecção do método principalmente em alguns meses do período estiagem e valor máximo de 2,73 $\mu \mathrm{mol}^{-\mathrm{L}^{-1}} \mathrm{em}$ julho/11 na estação 2 na superfície. O nitrito variou significativamente entre os períodos climáticos $(p=0,035)$ com valores mais elevados no período chuvoso, porém não foram observadas variações significativas entre as estações de coleta $(p=0,362)$.

Assim como para o nitrito, as concentrações de nitrato também apresentaram valores abaixo do limite de deteç̧ão do método e na estação 2 em julho/11 na superfície foi registrado o valor máximo de $15,82 \mu \mathrm{mol} . \mathrm{L}^{-1}$. O nitrato variou significativamente entre os períodos climáticos $(p=0,003)$ com valores mais elevados no período chuvoso não sendo registradas variações entre as estações de coleta $(p=0,894)$.

Quanto ao fosfato, as concentrações mínimas e máximas foram registradas na estação 4 em fevereiro/11 $\left(0,06 \mu \mathrm{mol} . \mathrm{L}^{-1}\right)$ na camada de fundo e na estação $2 \mathrm{em}$ novembro/10 $(6,36$ $\left.\mu \mathrm{mol} . \mathrm{L}^{-1}\right)$ na superfície, respectivamente. Foi registrada variação significativa apenas entre estações $(p=0,003)$.

As concentrações de silicato variaram de um mínimo de $1,00 \mu \mathrm{mol} . \mathrm{L}^{-1}$ registrado na estação 1 , superfície no mês de novembro/10 a $88,60 \mu \mathrm{mol} . \mathrm{L}^{-1}$ na estação 2 superfície em julho/11, sem variação significativa entre os períodos climáticos $(p=0,074)$ e espacial $(p=0,201)$, mesmo que os valores no período chuvoso tenham sido sempre mais elevados(Tab. 1). 
Tabela 1 - Características ambientais da água na zona costeira adjacente ao Porto do Recife (PE).

\begin{tabular}{l|r|r|r|r|r|r|r|r}
\hline \multirow{2}{*}{ Variáveis } & \multicolumn{2}{|c|}{ Estação 1 } & \multicolumn{2}{c|}{ Estação 2 } & \multicolumn{2}{c}{ Estação 3 } & \multicolumn{2}{c}{ Estação 4 } \\
\cline { 2 - 9 } & MIN & MAX & MIN & MAX & MIN & MAX & MIN & MAX \\
\hline Profundidade $(\mathrm{m})$ & 8,40 & 11,70 & 6,50 & 13,50 & 7,00 & 10,60 & 3,50 & 9,50 \\
\hline Transparência $(\mathrm{m})$ & 1,40 & 3,80 & 0,50 & 2,00 & 0,60 & 3,00 & 1,10 & 6,00 \\
\hline Salinidade & 24,76 & 36,63 & 14,80 & 36,34 & 15,34 & 37,16 & 27,99 & 37,16 \\
\hline $\mathrm{OD} \mathrm{ml} / \mathrm{L}^{-1}$ & 3,48 & 4,91 & 1,73 & 7,78 & 3,05 & 4,67 & 3,71 & 5,02 \\
\hline $\mathrm{MPS}\left(\mathrm{mg}^{-1} \mathrm{~L}^{-1}\right)$ & 3,80 & 48,94 & 8,80 & 57,00 & 5,00 & 64,00 & 2,80 & 44,68 \\
\hline $\mathrm{pH}$ & 8,01 & 8,76 & 7,97 & 8,94 & 7,96 & 8,42 & 8,06 & 8,94 \\
\hline $\mathrm{NO}_{2}\left(\mu \mathrm{mol} / \mathrm{L}^{-1}\right)$ & 0,00 & 2,28 & 0,00 & 2,73 & 0,00 & 2,33 & 0,00 & 0,85 \\
\hline $\mathrm{NO}_{3}\left(\mu \mathrm{mol} / \mathrm{L}^{-1}\right)$ & 0,30 & 6,77 & 0,31 & 15,82 & 0,36 & 14,61 & 0,00 & 9,02 \\
\hline $\mathrm{PO}_{4}\left(\mu \mathrm{mol} / \mathrm{L}^{-1}\right)$ & 0,07 & 1,02 & 0,10 & 6,36 & 0,23 & 3,17 & 0,06 & 1,20 \\
\hline $\mathrm{SiO}_{2}\left(\mu \mathrm{mol} / \mathrm{L}^{-1}\right)$ & 1,00 & 61,78 & 1,80 & 88,60 & 2,00 & 61,82 & 1,20 & 51,39 \\
\hline
\end{tabular}

\section{Composição taxonômica}

O microfitoplâncton foi representado por 123 táxons, pertencentes aos filos Ochrophyta, Myzozoa, Chlorophyta e Cyanobacteria (Fig.2). O filo Ochrophyta, particularmente a classe Bacillariophyceae, foi o mais abundante, representando $70,7 \%$ do total de organismos, com 87 táxons. As famílias de maior destaque foram: Chaetocerotaceae representada por 14 espécies, Triceratiaceae com 10 espécies, Rhizosoleniaceae, Bacillariaceae e Surirellaceae com seis espécies e Biddulphiaceae e Fragilariaceae com cinco espécies. As demais famílias estiveram representadas por menos de quatro espécies. Os gêneros que mais se destacaram foram Chaetoceros (14 espécies), Odontella e Nitzschia (quatro espécies); Biddulphia, Hemiaulus, Triceratium, Gyrosigma, Campylodiscus, e Surirella (três espécies). Os demais gêneros tiveram menos de duas espécies.

O filo Myzozoa constituiu o segundo grupo mais representativo com $15,4 \%$ do total de organismos, 19 táxons e apenas uma classe: Dinophyceae. As famílias desse filo foram: Dinophysaceae ( 2 espécies), Protoperidiniaceae (3 espécies), Podolampadaceae (1 espécie), Prorocentraceae (1 espécie), Ceratiaceae (10 espécies), Ceratocoryaceae (1 espécie) e Pyrophacaceae (1 espécie).

Foram identificadas 10 espécies de Chlorophyta, representando 8,1\% do total de organismos, com representantes de três classes (Chlorophyceae, Trebouxiophyceae e Zygnematophyceae), sete famílias (Volvocaceae, Chlorellaceae, Scenedesmaceae, Hydrodictyaceae, Closteriaceae, Desmidiaceae e Chaetophoraceae) e sete genêros (Eudorina, Actinastrum, Scenedesmus, Pediastrum, Closterium, Staurastrum e Stigeoclonium). As famílias com maior número de espécies foram Scenedesmaceae, Hydrodictyaceae e Desmidiaceae com duas espécies cada.

O filo Cyanobacteria esteve constituído por sete espécies, representado por 5,7\% do total de organismos, pertencentes a uma única classe (Cyanophyceae), duas ordens (Oscillatoriales e Pseudanabaenales), 3 famílias (Oscillatoriaceae, Phormidiaceae, Pseudanabaenaceae) e cinco gêneros (Oscillatoria, Phormidium, Planktothrix, Trichodesmium e Spirulina). A família Oscillatoriaceae foi a melhor representada, com três espécies (Fig.2). 


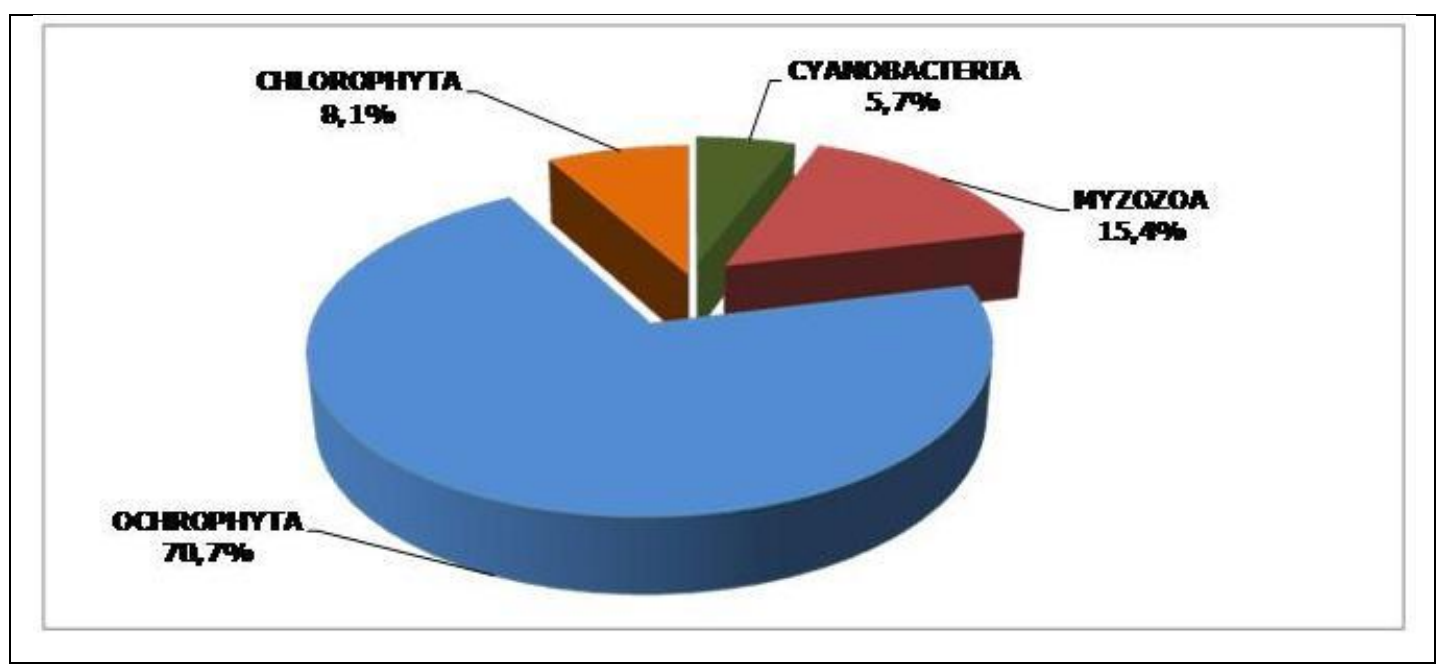

Figura 2 - Participação relativa dos principais filos do fitoplâncton identificados na zona costeira do Porto do Recife-PE.

\section{Abundância e frequência de ocorrência}

As diatomáceas destacaram-se como dominantes nos períodos de estiagem e chuvoso. No período chuvoso Chaetoceros curvisetus Cleve representou mais de $90 \%$ do total de organismos na estação 4 (março/2011) e Coscinodiscus centralis Ehrenberg representou mais de $80 \%$ dos organismos nas estações 1,3 e 4 (maio/2010). No período de estiagem destacaram-se Helicotheca tamesis (Shrubsole) M. Ricard nas estações 2, 3 e 4 (fevereiro/2011) com percentuais acima de $70 \%$ e Skeletonema cf. costatum (Greville) Cleve com mais de $82 \%$ do total de organismos nas estações 1, 2 e 3 (novembro/2010). Com relação à frequência de ocorrência, ainda neste grupo, foram muito frequentes: Asterionellopsis glacialis (Castracane) Round, Coscinodiscus centralis e Fragilaria capucina Desmazières. Entre as cianobactérias destacaram-se Planktothrix agardhii (Gomont) Anagnostidis \& Komárek em julho/2010 nas estações 2 e 3 com percentuais acima de $80 \%$ e Trichodesmium thiebautiiGomont ex Gomont em julho/2011 na estação 4, com percentual acima de 70\%, ambas dominando no período chuvoso.

\section{Riqueza}

A riqueza não variou significativamente entre os períodos climáticos $(p=0,757)$ e estações de coleta $(p=0,421)$. Observou-se, no entanto, que os valores foram sempre mais elevados no período de estiagem, e mínimos nas estações 2 e 3, independente do período climático (Fig.3). 


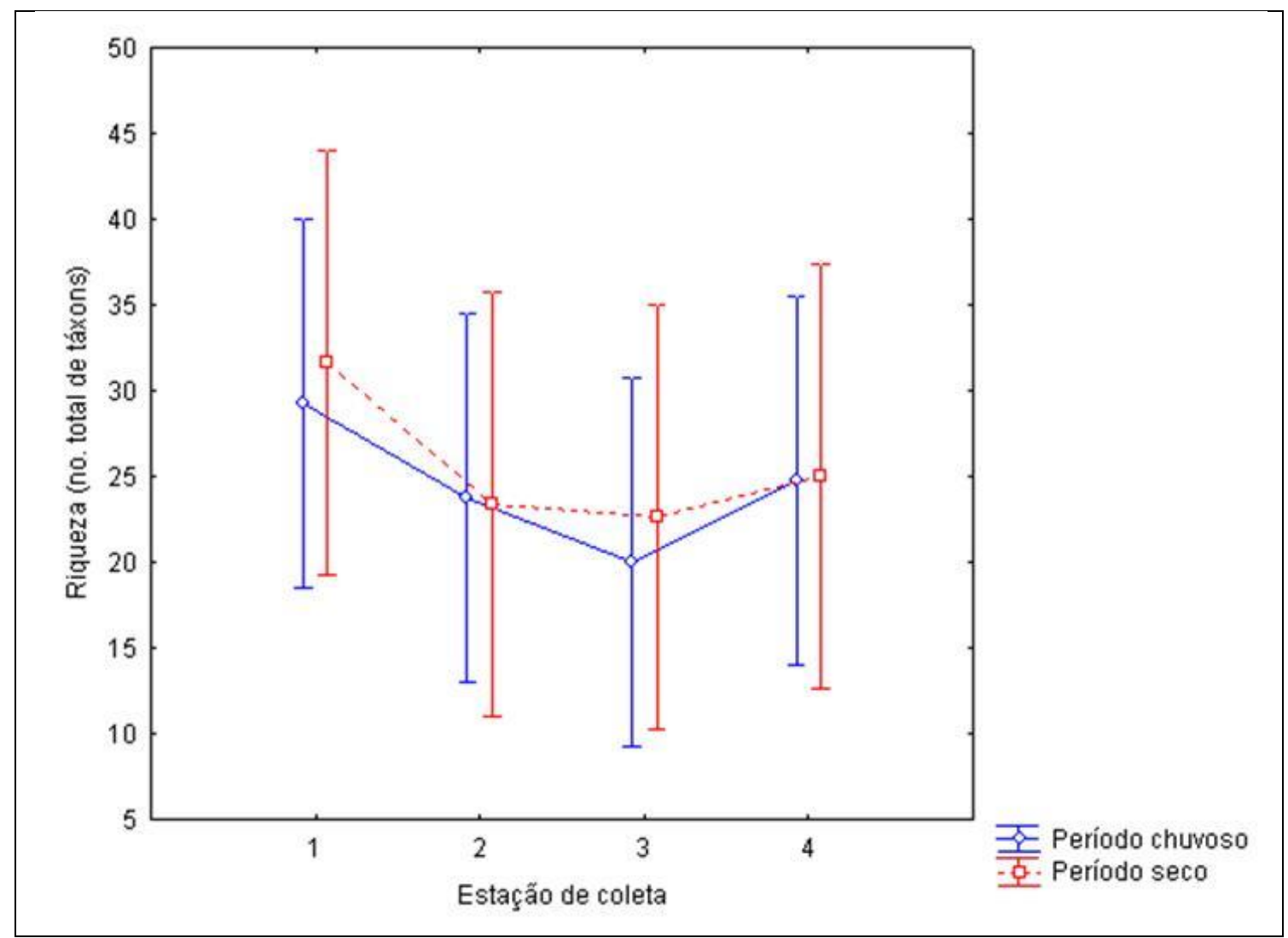

Figura 3 - Riqueza do fitoplâncton na zona costeira adjacente ao porto do Recife-PE.

\section{Diversidade e Equitabilidade}

A diversidade e a equitabilidade não variaram significativamente entre estações de coleta ou períodos climáticos (Figs. 4 A e B). A diversidade variou de 0,43 na estação 4 em março/2011 a 4,41 na estação 1 em julho/2010. Por sua vez, a equitabilidade foi mínima $(0,06)$ na estação 4 em março/2011 e máxima $(0,63)$ na estação 1 em julho/2010 (Fig. 4). 

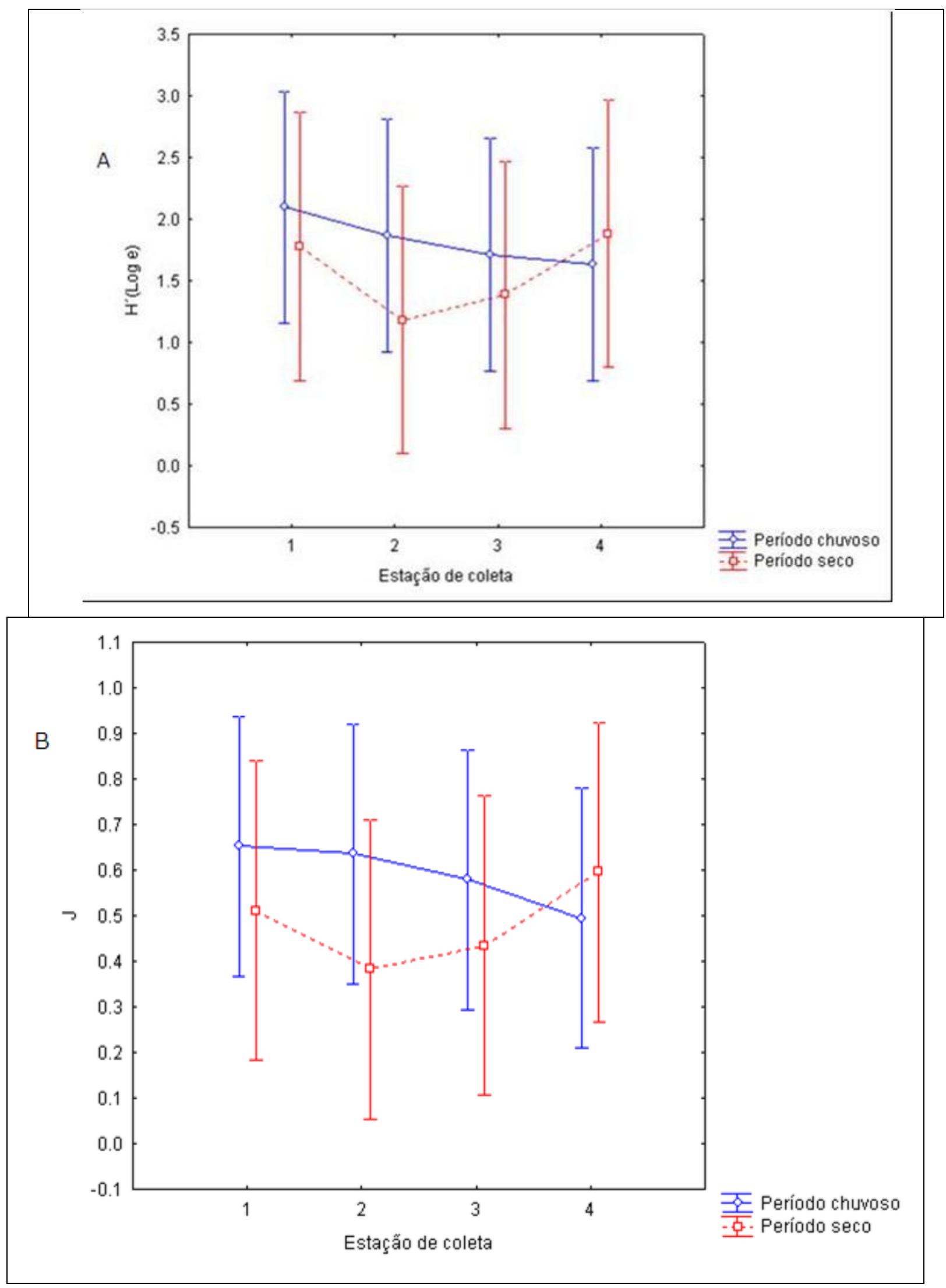

Figura 4 - Diversidade (A) e equitabilidade (B) do fitoplâncton na zona costeira adjacente ao porto do Recife-PE.

\section{Análise de Agrupamentos}

A associação das espécies permitiu evidenciar dois grupos principais: 
Grupo 1- composto por espécies marinhas planctônicas neríticas e características principalmente do período de estiagem.

Grupo 2 - mais diversificado taxonomicamente e com o predomínio de espécies frequentes e muito frequentes. Ecologicamente representado por espécies marinhas planctônicas neríticas, marinhas planctônicas oceânicas e espécies ticoplanctônicas.

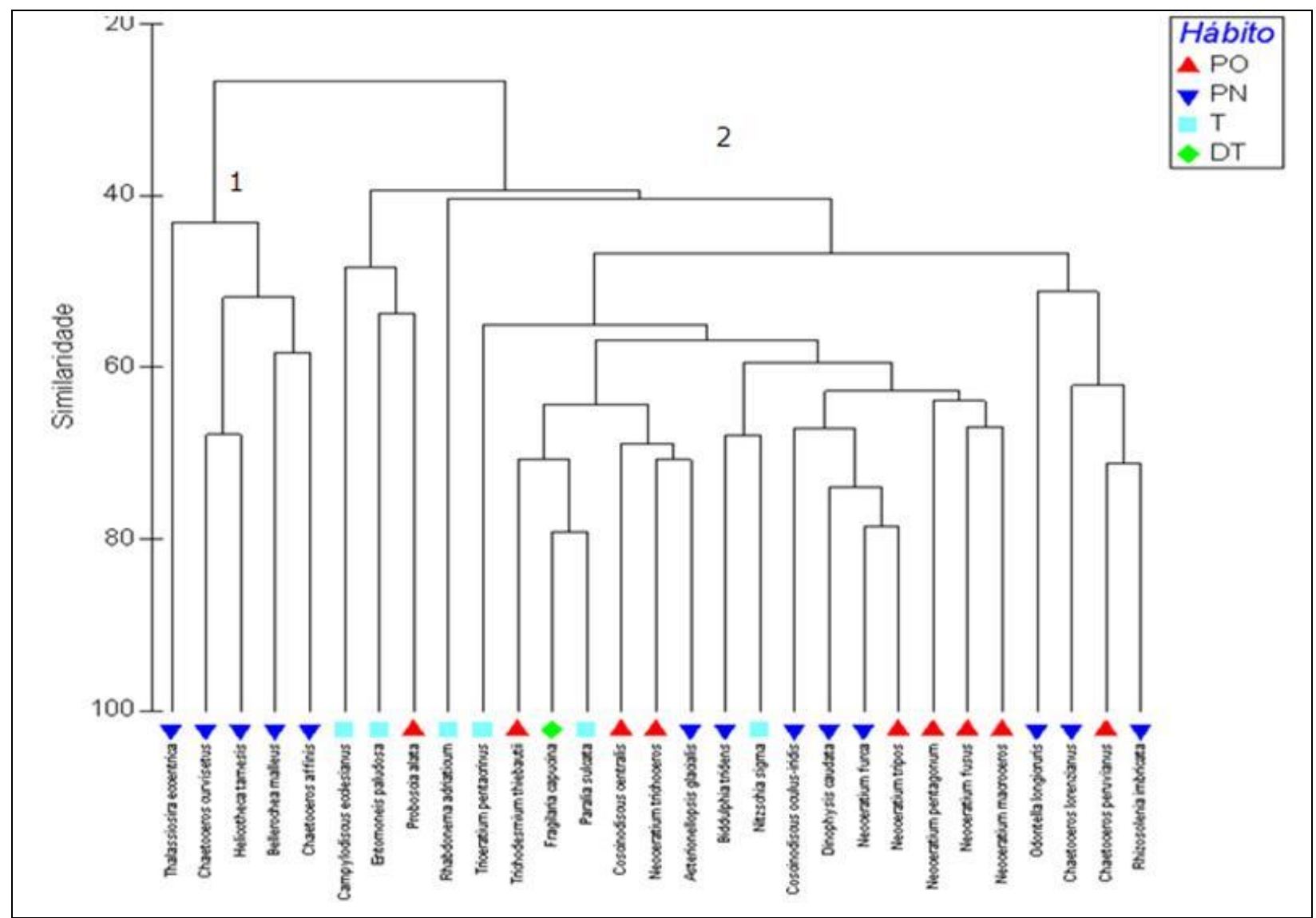

Figura 5 - Dendrograma dos agrupamentos formados pela associação das espécies mais representativas na zona costeira adjacente ao porto do Recife-PE.

\section{DISCUSSÃO}

A zona costeira segundo Miranda et al. 2002 é definida como a parte da plataforma continental que está sob a influência direta da massa de água estuarina e Trochimczuk e Schettini (2003) reforçam que a concentração de material particulado em suspensão e a proporção de água doce na pluma costeira são proporcionais à amplitude da descarga fluvial.

Trabalhos sobre as propriedades físicas das águas costeiras no litoral do estado de Pernambuco são poucos se destacando os trabalhos de Rollnic, (2002 e 2008) que estudaram a hidrologia e circulação da zona costeira da região metropolitana de Recife (PE). Os referidos estudos demonstraram a presença sazonal de uma pluma estuarina de baixa salinidade $(<33)$ na região costeira em torno da região metropolitana de Recife, associados às descargas de águas provindas dos rios Beberibe, Capibaribe e da Barra das Jangadas. Os maiores valores de salinidade superficial foram observados nas coletas realizadas durante o período de estiagem, devido provavelmente à ação da evaporação intensa e a redução de precipitação neste período do ano na região.

A área de estudo fica localizada em frente ao Porto do Recife sendo separada do oceano Atlântico por um molhe artificial de pedras e de blocos de concreto construído sobre um dique natural de recifes de arenito calcário e pelo extenso quebra mar paralelo à costa, localmente 
conhecido como molhe do inglês, que guarda a sua principal entrada (SÁ et al. 2011). A presença de recifes na zona costeira pode ser segundo Miranda et al. (2002) um obstáculo para as trocas de água entre o estuário e a região costeira.

Neste trabalho, das quatro estações estudadas, a estação 2 mostrou estar sujeita a uma influência direta da descarga dos estuários dos rios Capibaribe e Beberibe no primeiro metro da coluna d'água. O aporte destes rios determinou a extensão da pluma e como resultado, esta estação apresentou condições peculiares no que diz respeito às características físicas e químicas. Os valores de oxigênio dissolvido, profundidade e salinidade apresentaram valores mínimos, entretanto, foi registrado um aumento dos valores de turbidez, material em suspensão e sais nutrientes.

Estudos sobre a pluma em regiões costeiras e oceânicas indicam que esta pode causar um efeito sobre a ecologia regional de um ambiente, associado com o descarregamento de grandes quantidades de nutrientes e de material sedimentar na coluna de água, que são distribuídos pelas correntes marinhas e transportados ao longo da costa (MULLER-KARGER et al., 1995; HERRERA e BONE, 2011).

Estatisticamente, os parâmetros físicos e químicos analisados variaram espacial e sazonalmente de forma distinta. A maioria deles apresentou variação significativa apenas entre períodos sazonais, com exceção da profundidade salinidade e oxigênio dissolvido que variaram também entre as estações como registrado acima.

Apesar da transparência e material particulado em suspensão serem fatores limitantes para a comunidade fitoplanctônica (CLOERN et al. 1985), estes fatores não apresentaram na área estudada, variação significativa espacial ou em função dos períodos climáticos pela influência marinha na área,corroborando com os resultados obtidos por Honorato da Silva et al. (2009) para o estuário do rio Serinhaém e Santiago et al. (2010) para a bacia portuária do Recife.

Nas áreas costeiras de Pernambuco, mudanças temporais na distribuição e abundância do fitoplâncton são reguladas principalmente pela eutrofização, descarga dos rios, ciclo de maré e precipitação (ESKINAZI-LEÇA et al. 1997; FERREIRA et al. 2010). Um dos principais fatores que controlam a composição das espécies e o surgimento de florações é a quantidade dos principais elementos nutrientes (ANDERSON et al. 2002).

Durante o estudo uma grande quantidade de nutrientes foi registrada principalmente nas estações 2 e 3, contribuindo para o aumento da diversidade das diatomáceas $(70,7 \%)$ que dominaram em ambos os períodos sazonais. Segundo Siqueira (2003) quanto mais baixa a salinidade maiores são os níveis de sílica, uma vez que a sílica é oriunda de água doce, fato esse também observado na área estudada. Segundo Braga et al., 2009, altos valores de silicato normalmente estão associados com aportes terrestres, embora atuem como nutriente principal ao desenvolvimento do fitoplâncton. O silicato representa um constituinte importante para identificação de plumas em águas superficiais.

Para Procopiak et al. (2006) a presença das diatomáceas em áreas costeiras está condicionada às suas características de eurialinidade e condições eutróficas.

A dominância das diatomáceas como a entidade taxonômica mais representativa, é um fato já comumente registrado para as águas costeiras do Nordeste do Brasil (ESKINAZI-LEÇA et al. 2004; ROSEVEL DA SILVA et al. 2005 e SANTIAGO et al. 2010).

Outro fator que favoreceu o crescimento das diatomáceas foi a aparente redução das condições de luz, uma vez que as espécies deste grupo são caracterizadas por baixos requerimentos de luz e adaptadas às flutuações na intensidade de luz induzidas pela turbulência(SMAYDA e REYNOLDS, 2001).

$\mathrm{Na}$ área de estudo deve-se registrar a ocorrência de espécies dominantes como Chaetoceros curvisetus (90\%) e Coscinodiscus centralis $(80 \%)$ durante o período chuvoso e Helicotheca tamesis (70\%)e Skeletonema cf. costatum (82\%), durante o período de estiagem. Travassos (1991) estudando o estuário do rio Capibaribe registrou a ocorrência de Skeletonema 
costatum como espécie dominante e frequente cujos florescimentos foram registrados para o período de estiagem fato corroborado também no presente estudo.

Os resultados obtidos corroboraram com os de Santiago et al. (2010), em coletas realizadas em um ponto fixo na confluência do Porto do Recife, rio Capibaribe e o Pina (parte mais interna) que registraram o grupo das diatomáceas como predominante na área. Dentre as espécies dominantes, estes autores observaram também a Helicotheca tamesis característica do período de estiagem e Coscinodiscus centralis do período chuvoso. Resultados similares foram obtidos por Rosevel Silva et al. (2005) na Baía de Tamandaré e por Ferreira et al. (2010) em praias urbanas do litoral de Pernambuco. As referidas espécies são mencionadas na literatura como não tóxicas, embora potencialmente nocivas (Fernandes et al., 2001; Odebrecht et al., 2002; Procopiak et al., 2006; Proença e Fernandes, 2004).

Além das diatomáceas, no período chuvoso foram registradas algumas espécies dominantes de cianobactérias como Planktothrix agardhii (80\%) e Trichodesmium thiebautii (70\%). Estas espécies caracterizam a existência de problemas locais de eutrofização e condicionaram a uma baixa diversidade específica. São respectivamente citadas como formadoras de florações em reservatórios brasileiros e plataforma continental e potencialmente tóxicas (Sant'Anna et al., 2008; Monteiro et al., 2010).

Os resultados obtidos demonstram se encontrar o ecossistema livre de grandes impactos ambientais e preservado de suas características ecológicas, apesar das pressões urbanas e o desenvolvimento industrial terem contribuído para a eutrofização. Este processo acarretou a ocorrência no período chuvoso de florações de Planktothrix agardhii e Trichodesmium thiebautii, espécies consideradas potencialmente tóxicas e nocivas, indicando a necessidade de medidas de controle da qualidade da água do ecossistema.

\section{AGRADECIMENTOS}

Os autores agradecem ao Conselho Nacional de Desenvolvimento Científico e Tecnológico - CNPq, pelo auxílio financeiro ao projeto (Processo no: 558106/2009-9), ao Prof. Manuel de Jesus Flores Montes, coordenador do projeto pela concessão dos dados abióticos e ao Prof. Dr. Pedro Luiz Guzzo pelos comentários e sugestões.

\section{REFERÊNCIAS}

ANDERSON, D. M.; GLIBERT, P.M.; BURKHOLDER, J.M. Harmful algal blooms and eutrophication: nutrient sources, composition, and consequences. Estuaries, v. 25, p. 704 726, 2002.

ARAGÃO, J. O. R. A influência dos oceanos Pacífico e Atlântico na dinâmica do tempo e do clima do Nordeste do Brasil. In: ESKINAZI-LEÇA, E.; NEUMANN-LEITÃO, S.; COSTA, M. F. (Org.).

Oceanografia - Um cenário tropical. Recife: Bagaço, 2004. p. 287-317.

BRAGA, E. S.; ESCHRIQUE, S. A.; BASTOS, A. T. C. C.; COELHO, L. H. F.. Silicato dissolvido e seu papel traçador de aportes terrestres/sedimentares e sistemas estuarinos. In: XII Congresso Brasileiro de Geoquímica e VIII International Symposium on Environmental Geochemistry, 2009, Ouro Preto. XII Congresso Brasileiro de Geoquímica e VIII International Symposium on Environmental Geochemistry, 2009. v. 1. p. 1-4

COMPANHIA PERNAMBUCANA DO MEIO AMBIENTE (CPRH). Diagnóstico Sócio-Ambiental: Litoral Norte. Recife: CPRH/GERCO, 2001, p. 74-76.

CLOERN, J. E.; COLE B. E.; WONG, R. L. J., ALPINE, A. E. Temporal dynamics of estuarine phytoplankton: a case study of San Francisco bay. Hydrobiologia, v. 129, p. 153-176, 1985.

ESKINAZI-LEÇA, E.; KOENING, M. L.; SILVA-CUNHA. M. G. G. Estrutura e dinâmica da comunidade fitoplanctônica. In: ESKINAZI-LEÇA, E.; NEUMANN-LEITÂO, S.; COSTA, M. F. (Org.) Oceanografia: Um Cenário tropical. Recife: Bagaço, 2004. p. 353-373.

ESKINAZI-LEÇA, E.; SILVA-CUNHA, M. G. G.; KOENING, M. L.; MACEDO, S. J.; COSTA, K. M. P. Variação espacial e temporal do fitoplâncton na Plataforma Continental de Pernambuco- Brasil. Trabalhos Oceanográficos da Universidade Federal de Pernambuco, v. 25, p. 1-16, 1997. 
FEITOSA, F. A. N.; PASSAVANTE, J. Z. O. Variação sazonal da produção primária do fitoplâncton da Bacia do Pina (Pernambuco - Brasil). Trabalhos Oceanográficos da Universidade Federal de Pernambuco, v. 22, p. 65-82, 1991/1993.

FEITOSA, F. A. N.; NASCIMENTO, F. C. R.; COSTA, K. M. P. Distribuição espacial e temporal da biomassa fitoplanctônica relacionada com parâmetros hidrológicos na bacia do Pina (Recife-PE). Trabalhos Oceanográficos da Universidade Federal de Pernambuco, v. 27, n. 2, p.1-13, 1999.

FERREIRA, L. C.; SILVA-CUNHA, M. G. G. KOENING, M. L.; FEITOSA, F. A. N.; SANTIAGO, M. F.; MUNIZ, K. Variação temporal do fitoplâncton em três praias urbanas do litoral sul do estado de Pernambuco, Nordeste do Brasil. Acta Botanica Brasilica, v. 24, n. 1, p. 214-224, 2010.

FERNANDES, L. F.; ZEHNDER-ALVES, L.; BASSFELD, J. The recently established diatom Coscinodiscus wailesii (Coscinodiscales, Bacillariophyta) in Brazilian waters. In: remarks on morphology and distribution. Phycological Research, v. 49, p. 89-96, 2001.

GRASSHOFF, K.; EHRHARDT, M.; KREMLING, K. (Ed.). Methods of seawater analisys. 2. ed. Verlag Chemie, 1983. 419 p.

HERRERA, A.; BONE, D. Influence of riverine outputs on sandy beaches of Higuerote, central coast of Venezuela. Latin American Journal Aquatic Research, v. 39, n.1, p. 55-70, 2011.

HONORATO DA SILVA, M. Estrutura e produtividade da comunidade fitoplanctônica de um estuário tropical (Sirinhaém, Pernambuco, Brasil). Recife, 2009, $170 \mathrm{f}$. Tese (Doutorado em Oceanografia). Departamento de Oceanografia. Universidade Federal de Pernambuco.

KOENING, M. L.; MACÊDO, S. J.; TRAVASSOS, P. E. P. F.; PASSAVANTE, J. Z. O. Biomassa fitoplanctônica no estuário do rio Capibaribe (Recife - Pernambuco -Brasil). Arquivos de Biologia e Tecnologia. v. 38, n. 4, p. 1071-1083, 1995.

LIVINGSTON, R. J. Eutrophication processes in coastal systems: origin and succession of plankton blooms and effects on secondary production in Gulf Coast Estuaries Center for Aquatic Research and Resource Management. Florida State University, CRC Press, 2001, 327 p.

LOBO, E.; LEIGHTON, G. Estructuras comunitárias de las fitocenosis planctonicas de lós sistemas de desembocaduras de rios y esteros de la zona central de Chile. Revista Biológica Marina y Oceanografia, [s.i.]., n. 22, p. 1-29, 1986.

MATEUCCI, S. D.; COLMA, A. La metodologia para el estudo de la vegetacion. Collection de Monografias Cientificas, [s.i.]., n. 22, 1982, 168 p. (Serie Biologia).

MELO, V.; SUMMERHAYES, C. D.; TONER, L. G. Metodologia para estudos do material em suspensão na água do mar. Boletim Técnico da Petrobrás, v. 18, n. 314, p. 115-127, 1975.

MIRANDA, R.; CHIAN, A.; KOGA, D.; REMPEL, E.; SAIKI, Y.; YAMADA, M. Amplitude-phase synchronization in intermittent turbulence and spatiotemporal chaos. In: Dynamics days South America 2010: International Conference On Chaos And Nonlinear Dynamics, 2010, São José dos Campos. Proceedings... São José dos Campos: INPE, 2010. On-line. Disponível em: http://urlib.net/8JMKD3MGP7W/382LDGL.

MIRANDA, L. B.; CASTRO, B. M.; KJERFVE, B. Princípios de oceanografia física de estuários. São Paulo: EDUSP, 2002. 411 p.

MONTEIRO, L. H. U.; SILVA, G. S.; LIMA, L. L. L.; COUTINHO, P. N. Estudos hidrodinâmicos e hidroquímicos do estuário do rio Capibaribe e Bacia do Pina, Recife - PE, V Simpósio

Brasileiro de Oceanografia - Oceanografia e Políticas Públicas, Santos, SP, Brasil - 2011.

MULLER-KARGER, F. E.; RICHARDSON, P. L.; MCGILLICUDDY, D. J. On the offshore dispersal of the Amazon's Plume in the North Atlantic. Deep-Sea Research I, v.42, n.11/12, 2127-2137, 1995. 
NASCIMENTO, F. C. R.; MUNIZ, K.; FEITOSA, F. A. N.; ARAÚJO, J. P. de; SILVA, R. M. S. da; SÔNIA DA SILVA, G.; FLORES MONTES, M. de J. Disponibilidade nutricional da Bacia do Pina e Rio Tejipió (Recife-PE-Brasil) em relação aos nutrientes e biomassa primária (Setembro/2000). Tropical Oceanography, v. 31, n. 2. p. 149-169, 2003.

NIXON S. W. Coastal marine eutrophication: a definition, social causes, and future concerns. Ophelia, v. 41, p. 199-219, 1995.

ODEBRECHT, C.; AZEVEDO, S. M. F. O.; GARCIA, V. M. T.; HUSZAR, V. L. M.; MAGALHÃES, V. F.; MENEZES, M.; PROENÇA, L. A. O.; RÖRIG, L. R.; TENENBAUM, D. R.; VILLAC, M. C.; YUNES, J. S. Floraciones de microalgas nocivas en Brasil: estado del arte y proyectos en curso. In: Floraciones algales nocivas en el Cono Sur Americano (E.A. Sar, M.E. Ferrario \& B Reguera, eds). Instituto Español de Oceanografia, p.217-233, 2002.

OLIVEIRA FILHO, J. D. de. Adequação da gestão ambiental do porto do Recife à ISO 14.001:2004. Monografia de MBA Planejamento e Gestão Ambiental. Recife: FCAP/UPE, 2008.

PAERL, H. W.; VALDES, L. M.; PINCKNEY, J. L., PIEHLER, M. F.; DYBLE, J.; MOISANDER, P. H. Phytoplankton photopigments as indicators of estuarine and coastal eutrophication.

BioScience, v. 53, p. 953-964, 2003.

PIELOU, E. C. Mathematical ecology. New York: Wiley. 1977, 385 p.

PROCOPIAK, L. K.; FERNANDES, L. F.; MOREIRA-FILHO, H. Diatomáceas (Bacillariophyta) marinhas e estuarinas do Paraná, Sul do Brasil: lista de espécies com ênfase em espécies nocivas. Biota Neotropica, v. 6, n. 3.1-27, 2006.

PROENÇA, L. A. O.; FERNANDES, L. F. Introdução de microalgas no ambiente marinho: impactos negativos e fatores controladores. In: Água de lastro e bioinvasão. SILVA, J. S. V.; SOUZA, R. C. C. L. (eds). Editora Interciência, Rio de Janeiro, p.1-224, 2004.

ROLLNIC, M. Hidrologia, clima de onda e transporte advectivo na zona costeira de Boa Viagem, Piedade e Candeias - PE. Recife, 2002. 111 f. Dissertação (Mestrado em Oceanografia) Departamento de Oceanografia. Universidade Federal de Pernambuco.

ROLLNIC, M. Dinâmica das ondas e circulação no litoral da região metropolitana do Recife - implicações para o transporte de sedimentos e estabilidade da costa. Recife, 2008, 124 f. (Tese em Oceanografia). Departamento de Oceanografia. Universidade Federal de Pernambuco.

ROSEVEL DA SILVA, M.; SILVA-CUNHA, M. G. G.; FEITOSA, F. A. N.; MUNIZ, K. Estrutura da comunidade fitoplanctônica na baía de Tamandaré (Pernambuco, Nordeste do Brasil). Tropical Oceanography, v. 33, n. 2, p.163-181, 2005.

SÁ, M. E. M. de; LEAL NETO, A. de C.; FLORENCIO, L. Síntese da análise comparativa entre os portos do Recife e de Suape: desafios para a gestão ambiental. Tropical Oceanography,v. 39, n. 2. p. 1-16, 2011.

SANTIAGO, M. F.; SILVA-CUNHA, M. G. G.; NEUMANN-LEITÃO, S.; MUNIZ, K.; PALMEIRA, G. C. B.; NETO, F. F. P.; NUNES, F. S. Phytoplankton dynamics in a highly eutrophic estuary in tropical Brazil. Brazilian Journal of Oceanography, v. 58, n. 3, p. 189-205, 2010.

SCHETTINI, C. A. F.; KUROSHIMA, K. N.; PEREIRA FILHO, J.;RÖRIG, L. R.; RESGALLA JUNIOR, C. Oceanographic and ecological aspects of Itajaí-açu river plume during a high discharge period. Anais da Academia Brasileira de Ciências, v. 70, n. 2. p. 335-351, 1998.

SIQUEIRA, G. W. Distribuição de nutrientes inorgânicos dissolvidos e parâmetros físicos e físicoquímicos na região costeira Norte Brasileira. Rev. de Gestão Costeira Integrada para Países de Língua Portuguesa, v. 3, p.65-67, 2003.

SHANNON, C. E. A mathematical theory of communication. Boll. Syst. Tech. J.v. 27, p. 379423, 1948. 
SMAYDA, T. J.; REYNOLDS, C. S. Community assembly in marine phytoplankton: application of recent models to harmful dinoflagellate blooms. Journal of Plankton Research,v.23: 447461, 2001.

STRICKLAND, J. D. H.; PARSONS, T. R. A practical handbook of sea water analysis. 2. ed. Bulletin Fisheries Research Board of Canada, Ottawa, n. 167, p. 207-211, 1972.

TRAVASSOS, P. E. P. F. Hidrologia e biomassa primária do fitoplâncton do estuário do Rio Capibaribe, Recife - Pernambuco. Recife, 1991. 288 f. Dissertação (Mestrado) Departamento de Oceanografia. Universidade Federal de Pernambuco.

TROCHIMCZUK F. O. A.; SCHETTINI, C. A. F. Avaliação da dispersão espacial da pluma do estuário do rio Itajaí-Açu em diferentes períodos de descarga. Notas Técnicas da Facimar, v. 7, p. 83-96, 2003.

UNESCO. International Oceanographic Table.Great Britain Wormly, n. 2, 1973. 141 p. UNESCO. Background papers and supporting data on the practical salinity scale 1978, Technical Papers Marine Sc., vol. 37, 144 p. 1981. 